\title{
Model-Based Simulation System for Planning Numerical Controlled Multi-Axis 3D Surface Scanning Machine*
}

\author{
Peng WU**, Hiromasa SUZUKI ${ }^{* *}$ and Kiwamu KASE***
}

\begin{abstract}
This research proposes a model-based planning system for planning multi-axis 3D surface scanning machine. Firstly we describe a simulation system for performing collision avoidance, occlusion detection, and evaluating the measured portions of the surface. Then based on this simulation system, as our initial trial, we propose a simple path planning method to increase surface coverage and reduce measuring time by iterations. This research aims at measurement of complicated automotive parts, such as sheet metal parts and press dies. The implementation result shows the efficiency and automation of our approach.
\end{abstract}

Key Words: Model-Based Planning, Scanning, Simulation, Measurement

\section{Introduction}

3D surface scanning has been widely adopted with the development of numerically controlled scanning machines recently, it can be defined as the problem of finding the suitable sensor configuration such as viewing pose, camera focus and field of view, and etc. so that an inspection task can be carried out satisfactorily. According to whether geometrical information is available prior to the acquisition process or not, 3D surface planning can be divided into two classes basically. One class is the nonmodel-based planning that no geometrical information is available, which has been widely treated as a surface acquisition problem in the computer graphics community. Another class is the model-based planning that a complete model of the scene is known in advance, the plan can be computed off-line using the reference model. The related works about these two kinds of planning will be descried in section 2 .

Although in the past two decades plenty of researches for non-model-based planning have been developed, in the industry model-based planning for numerical controlled measuring system is becoming more and more essential in order to achieve efficient measurement results. However it is a fact that few model-based planning systems have

\footnotetext{
* Received 9th June, 2004 (No. 04-4152)

** Research Center for Advanced Science and Technology, The University of Tokyo, 4-6-1 Komaba, Meguro, Tokyo 153-8904, Japan. E-mail: wupeng@den.rcast.u-tokyo.ac.jp

*** The Institute of Physical and Chemical Research, 2-1 Hirosawa, Wako, Saitama 351-0198, Japan
}

been developed. The objective of this research is to develop an effective model-based simulation system, and to present a rough measurement planning for the multi-axis (3-axes, 4-axes and 6-axes) 3D scanning machine before actual measurement. We select our targets as car body structures, press die surfaces. The pre-generated scanning paths can be verified with visual simulation.

The remainder of this paper presents our research as follows: section 3 gives our planning system architecture. Section 4 presents our system simulation; section 5 presents a measurement planning including a path planning and sensor planning. Section 6 presents our implementation results. Section 7 gives our conclusion and describes the future work of fine planning.

\section{Related Works}

For non-model-based planning there is no complete geometrical information available prior to the acquisition phase, so the best way is to build up the plan on-line step by step. The planning is centralized in processing the known next-best-view (NBV) problem. The non-modelbased planning is categorized into three types: surfacebased method, volumetric method, and global view planning method. In the surface-based method J. Maver and R. Bajcsy took attention to an occlusion which arises either when the reflected laser light does not reach the camera or when the directed laser light does not reach the scene surface, and use the occlusion to indicate surface areas not yet sampled to guide the $\mathrm{NBV}^{(1),(2)}$ utilized contour following for guide the sensor to "paint" the object surface, and uncertainty is used to segment range data to guiding the sensor in Ref. (3). In volumetric method voxel occu- 
pancy grids and octrees are widely used to solve the NBV problem $^{(4),(5)}$. In global view planning method NBV decision is based on a global analysis of the mass vector chain for the object surface. The intermediate space technique ${ }^{(6)}$ has been used to separate visibility analysis of the object surface from that of the sensor.

For model-based planning, Refs. (7) and (8) provide a common scheme that employs the hypothesis-and-verify paradigm in the space of poses. Hypotheses are formed regarding the objects' identities and poses, and assessed in compliance to some metrics. New sensing operations are performed accordingly until a halting condition is met. F. Xia C. Shu proposed a CAD-based path planning for 3-D line laser scanning ${ }^{(9)}$ with a slicing approach to divide a part CAD model into a number of sections, then use each section to generate a scanning path. Although it shows its automation of scanning process, but the 3-D line laser scanning still have the low efficient problem. W.H. Sheng, et al. proposed a CAD model guided camera-planning system that utilized the CAD data of inspected parts and the camera model to plan camera viewpoints ${ }^{(10)}$ with active face-capturing sensor. But this algorithm depends on the topology property of multi-patches and cannot correspond to a general triangle mesh, and its tolerance is limited at several millimeter so that it is still not suited for precise part inspection possibly.

\section{System Architecture}

As shown in Fig. 1, the model-based planning system in this research consists of two major parts: model-based planner and scanning machine simulator. The modelbased planner takes the CAD model and the sensor model as two inputs to generate scanning paths. Once the scanning paths are determined, the NC-based controller that imports these codes and carries out the scanning task. The scanning simulator can execute not only motion simulation but also collision detection, occlusion detection, and capture simulation. Collision detection is to detect the collision between simulator and object surface, and occlusion detection is to detect if the directed laser light is prevented from other part and does not reach the object surface. With this capture simulation a number of scanned points data (position data) can be obtained.

\section{Simulation System}

We develop a simulator to simulate a 3D scanning machine functions. Subsection 4.1 describes the detail of the mechanical configuration model to be used for simulating the motion of the machine, and subsection 4.2 describes the sensor model used to simulate the sensor occlusions and position capture process. In subsection 4.3 we describe the capture simulation. Collision detection and occlusion detection are also processed and they are described in subsection 4.5 and 4.6 respectively.

\subsection{Mechanical link model for motion simulation}

The machine configuration consists of two parts. One is a mechanical manipulator and the other is a scanning sensor model. The mechanical manipulator is modeled as an open-loop articulated chain with rigid bodies (links). These links are connected in series by either rotational or linear joints, which are widely used in robotics ${ }^{(11)}$. One end of them manipulator is connected to a fixed platform; the other is attached with the scanning sensor. In this research we construct a manipulator with 6 DOF (degrees of freedom), which are three translation axes: $X, Y$ and $Z$-axes, and three rotation axes: $U, V$ and $W$, as shown in Fig. 2.

To each of the links connected by joints, we attach a

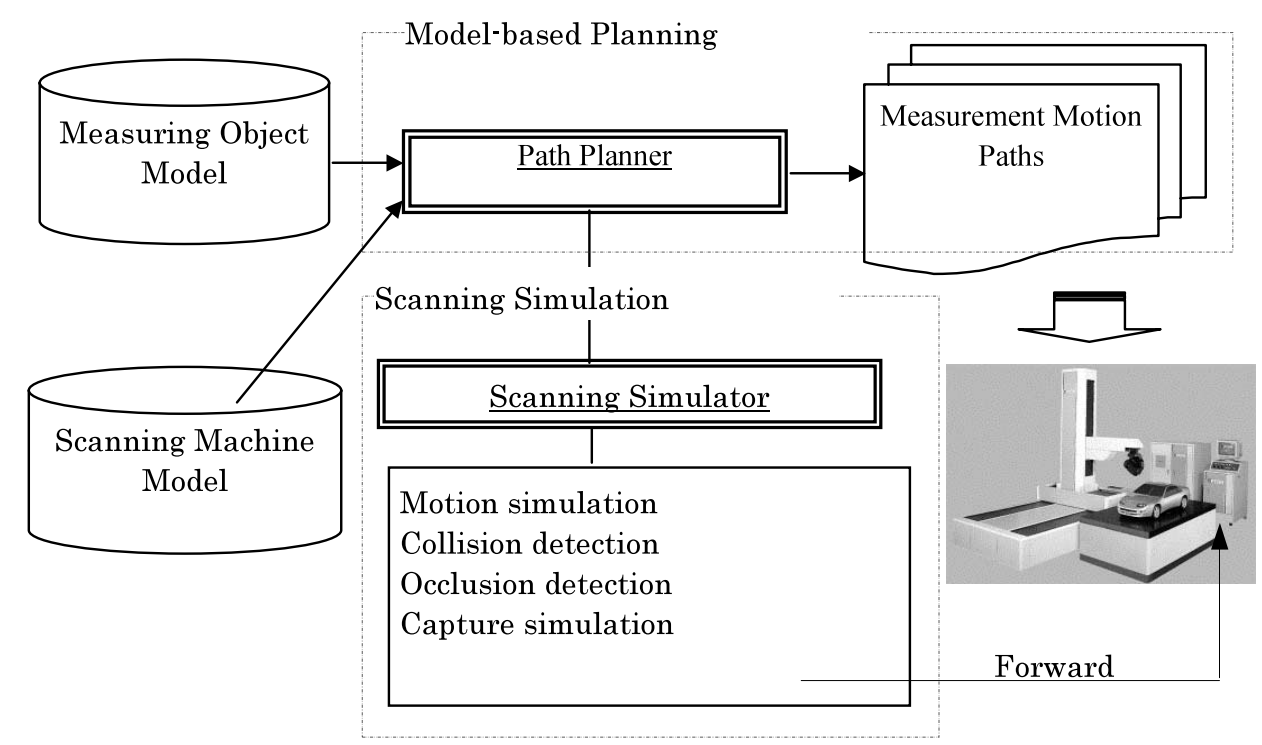

Fig. 1 Architecture of model-based planning system 


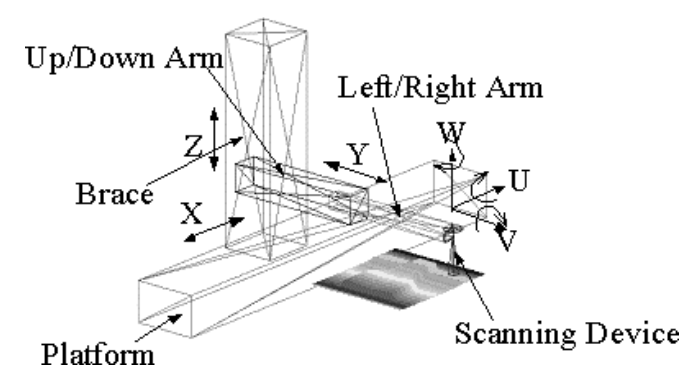

Fig. 2 Machine configuration with links/joints and scanning sensor model

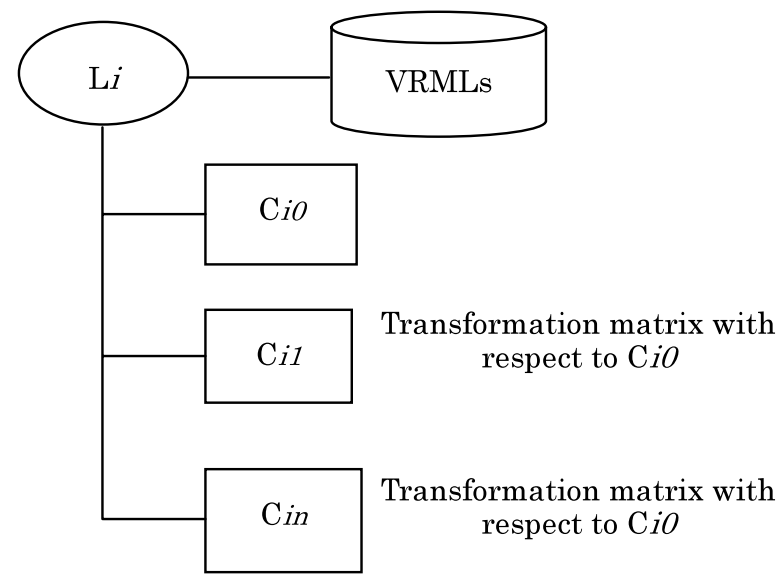

Fig. 3 Data structure of link

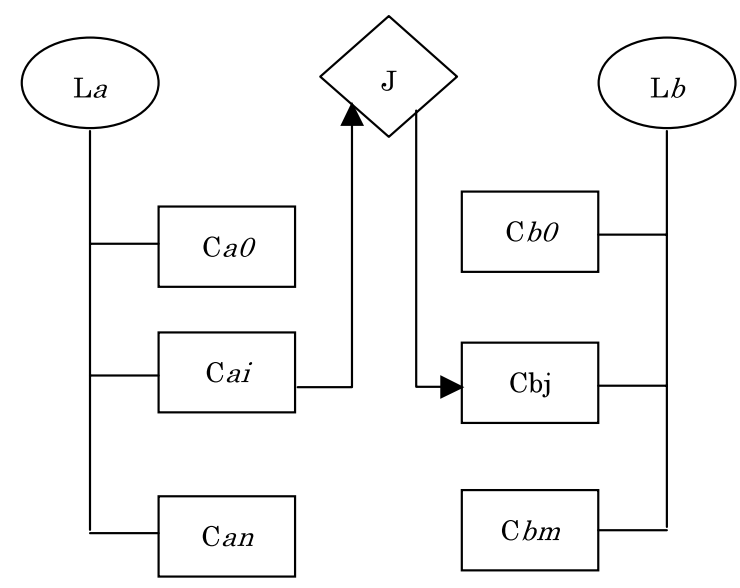

Fig. 4 Data structure of joint

local coordinate $C i 0$ and several relative coordinates $C i 1$, $\ldots, C$ in to each link $L i$, as shown in Fig. 3. The relationship between two links is described with a transformation matrix between their relative coordinates $\mathrm{Cai}$ and $\mathrm{Cbi}$, as shown in Fig. 4. The first matrix relates the link 0 to the base (platform Link), and the last matrix relates the last link to the sensor.

Two main problems of kinematics need to be dealt with in the simulation system:

One is forward kinematics that gives the joint angles or length of each link to find the location of the sensor. The other is inverse kinematics that gives the position of

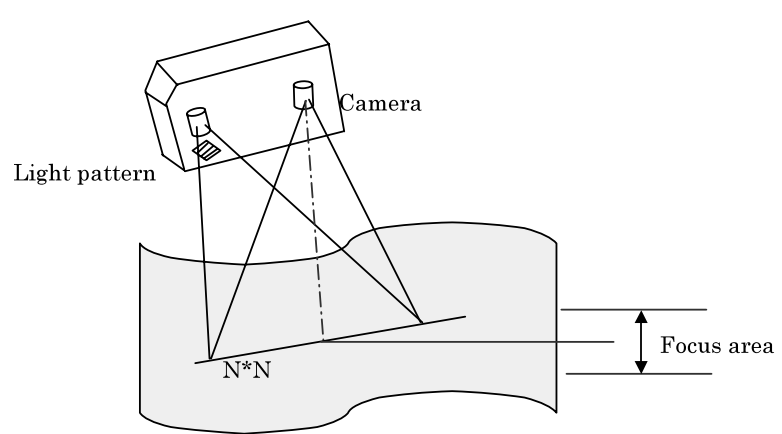

Fig. 5 A moiré sensor

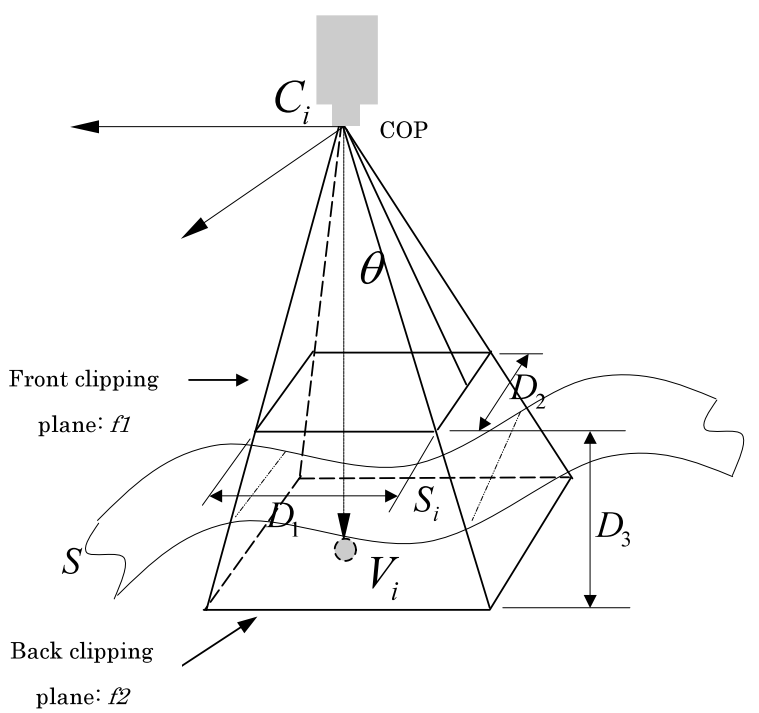

Fig. 6 Geometry of the sensor model

the sensor to find the joint displacements and angles.

All the shapes of the link models are represented with triangular meshes.

\section{2 Sensor model}

In this research we use a non-contact 3D active optical surface-scanning sensor, which obtains 3D coordinates by projecting specific light patterns on the surfaces of the target object. The 3D active optical sensor uses the moiré principle to capture the data clouds (see a moiré sensor Fig. 5), and it can capture the position data of $N \times N$ array of $3 \mathrm{D}$ points in a square area by one shot of scanning, where $N$ is decided by the specification of a sensor.

We pay attention to the similarities between the active sensor and a viewing model used in computer graphics ${ }^{(14)}$. The basic elements in both cases are the same. They have objects, a camera, and a projection plane. The projectors meet at the center of projection (COP). The COP corresponds to the center of the sensor of the scanning system and the center of the camera in the computer graphics systems. This gives us a convenient way to utilize the OpenGL hardware acceleration function in this research. Figure 6 describes the OpenGL geometry of the viewing volume equivalent to the real active sensor used in our re- 
search. We call it sensing volume hereafter. The sensing volume can be thought as a truncated pyramid. At the $i$-th scanning, we define the center of projection (COP) $C_{i}$ and two clipping planes, which are front clipping plane $f 1$ and back clipping plane $f 2$, and the sensor viewing direction $\vec{V}_{i}$ must be also assigned. The sensing volume $M_{i}=\left(C_{i}, \vec{V}_{i}\right)$ is defined by the two clipping planes $f 1$ and $f 2$, which can be defined by the distances from $C_{i}$ to them; $d_{1}$ for plane $f 1, d_{2}$ for $f 2$. Their values are fixed for the sensor model. Let $S$ be a surface of the target object, such portions of $S$ that intersect with the sensing volume are measured. The geometry of sensor model and object surface are also represented as triangle meshes in this research.

\section{3 Capture simulation}

The aims of capture simulation are two-fold. The first aim is to detect captured area on the object surface, which is described in subsection 4.3.1. The second aim is to compute measured data points, which is described in subsection 4.3.2.

4.3.1 Captured area detection As described in section 4.2, the surface of the target object is represented as a triangular mesh. We find a set of triangular faces captured by the sensor. Each triangular face of the mesh is given a unique T-ID (triangle ID) data. In order to realize the real-time enumeration of T-IDs for the captured triangular faces, we utilize a graphics library functions provided by a graphics hardware. Particularly, we use OpenGL hardware acceleration algorithm to capture the triangle IDs rapidly. In OpenGL the camera (scanning sensor) viewing volume is characterized by the location, orientation, projection of a camera and clipping planes. The basic idea of our method is to specify a viewing volume corresponding to the sensing volume according to the actual sensor's specification. Then we use OpenGL function to render the object surface (a triangular mesh), and get triangle IDs rendered by OpenGL camera, which correspond to those measured by the sensor.

In OpenGL one of the regular visualization processes is a geometrical part. The geometrical part converts all the coordinates of the scene polygons into the camera co-

ordinate system, clips all the faces relatively to the viewing volume, and achieves the perspective projection in order to get screen coordinates ${ }^{(12)}$. The geometrical part can help us to complete our capturing process. To realize this OpenGL provides the select mode GL_SELECT, which may be selected alternatively to the usual rendering mode GL_RENDER as the parameter of function glRenderMode(). Moreover because of no rasterization and lighting operation made at the select mode, the processing cost is very small. The select mode GL_SELECT provides information about T-IDs. A group name is given using glPushName() before each group of faces drawn, and OpenGL fills an array (provided by glSelectBuffer()) during the geometric part of rendering, writing an entry per group that appears in the viewing volume. Thus one can know the faces that appear on screen.

The following is the detail of implementation of TIDs capturing process, and Fig. 7 shows example result. Ahead of GL_SELECT operation we set up the COP of camera (scanning sensor) at an assigned position with gluLookAt(). The corresponding code is briefly listed in the following:

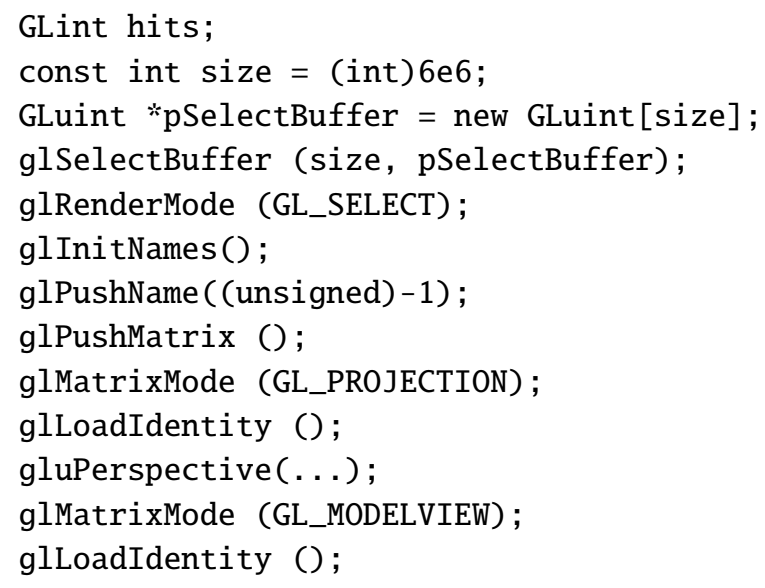

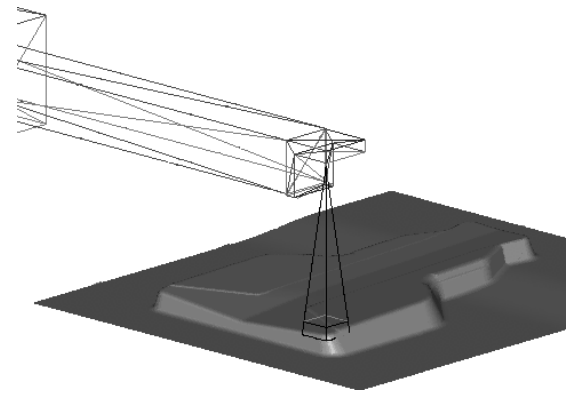

(a) Capture T-ID of the target object mesh

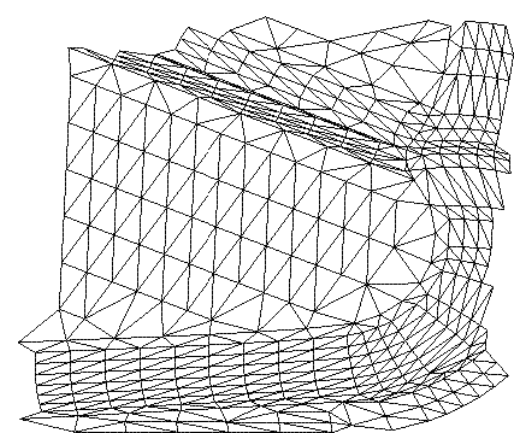

(b) T-IDs from the object surface with one shot

Fig. 7 T-ID data capture 
float eyex, eyey, eyez, centerx, centery, centerz, upx, upy, upz;

gluLookAt ( eyex, eyey, eyez, centerx, centery, centerz, upx, upy, upz);

\{

glLoadName (i) ;

: :glBegin(GL_TRIANGLES);

..........

glEnd () ;

\}

glPopMatrix();

glFlush ():

hits = glRenderMode (GL_RENDER); processHits (hits, pSelectBuffer); delete []pSelectBuffer;

4.3.2 Position data acquisition To capture position data we also use the OpenGL hardware acceleration. The hidden-surface-removal algorithm, which is also called z-buffer algorithm, is adopted in this research. Almost all the current 3D accelerated graphics hardware has been equipped this algorithm.

The basic idea of the z-buffer algorithm is shown in Fig. 8. When a 3D scene is rasterized with depth buffering enabled, at the beginning of the test, the depth value in the depth buffer is set to the largest possible value for the scene. The color value on the rendering surface is set to either the background color value. Each polygon in the scene is tested to see if it intersects with the current coordinate $(x, y)$ on the rendering surface. If it does, the depth

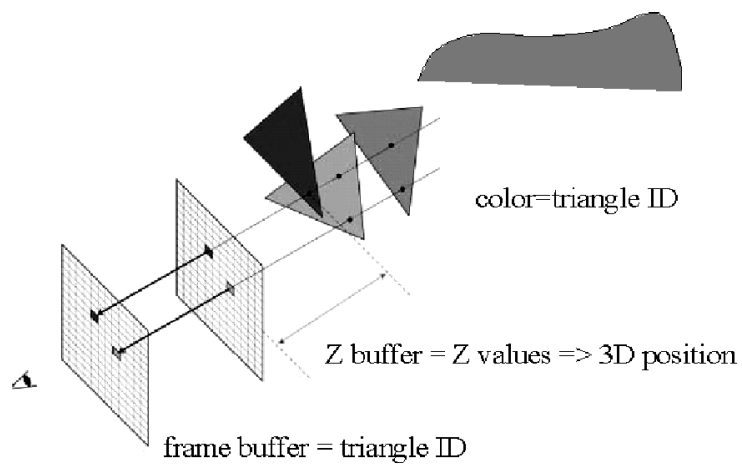

Fig. 8 The z-buffer algorithm

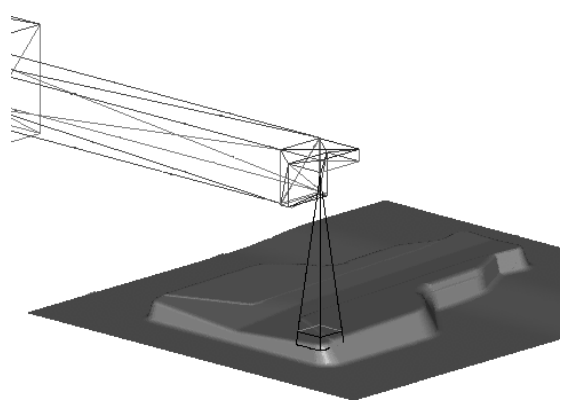

(a) Capture T-ID of the target object mesh value - which will be the $z$ coordinate in a z-buffer, and the $w$ coordinate in a $\mathrm{z}$-buffer is tested to see if it is smaller than the depth value stored in the depth buffer. Then its homogeneous location $(x, y, z, w)$ can be found. This z-buffer value can come from the standard memory in the system, or special memory can be added in at the end of a hardware pipeline.

In our system, by rendering the triangular mesh of the target object using the viewing volume defined in the previous section and also with the $N \times N$ pixels, positions on the surface are obtained. Figure 9 shows that a data position capturing with the z-buffer algorithm.

\section{4 Collision detection}

Although the motion simulation can directly give us the visualization of checking the collision, it is not enough only by visualization. Here we present a method of collision detection. About collision detection many previous works have been proposed in the past years ${ }^{(16)-(18)}$. In this research the main purpose of collision detection is to detect collision between the scanning machine links and target object. The target object is static while links are moveable.

The following two problems are considered in this research.

( i ) One is the calculation speed problem. The target object usually consists of large complex geometries composed of hundreds of thousands of triangles. It is also necessary for us to calculate the collision at real-time. So speed is an important issue.

(ii) The other is the accuracy problem. Following a motion path generated by inaccurate collision detection the real scanning machine can be damaged, even though there is safety protection function equipped with the scanning machines.

Among the recent approaches, those based on finding bounding volumes that tightly fit the object's geome-

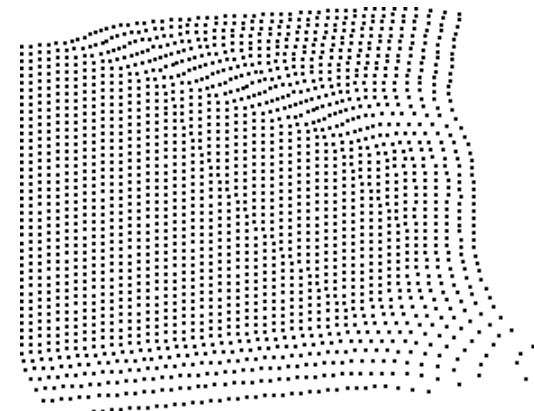

(b) Captured position data from the target object mesh with one shot

Fig. 9 Captured position data 
try are considered in this research. Gottschalk ${ }^{(13)}$ obtains very good results by using hierarchies of oriented bounding boxes instead of axis-aligned boxes. This Obb-tree approach meets our requirement well. With this approach we detect the collision between Links and the measuring object, and do not generate path at the collision occurred positions.

\subsection{Occlusion detection}

Because of the complex geometries of the target object, occlusion may occur between the sensor and the object. The occlusion occurs when a part of the target object lies between the sensor and the scanning volume. This part occludes the sensor from the other part of the object to be measured. From Fig. 10 we can see the region between the front clipping plane and back clipping plane cannot be captured well.

In order to detect the occlusion we give a simple method by moving the clipping planes of the OpenGL sensor model, as shown in Fig. 11, to detect if some objects exist between the front plane and the sensor. The previous front clipping plane is defined as the back clipping plane of occlusion OpenGL sensor model, and its front clipping plane is located at the COP. Then if some triangles are drawn in the occlusion OpenGL sensor model, occlusion

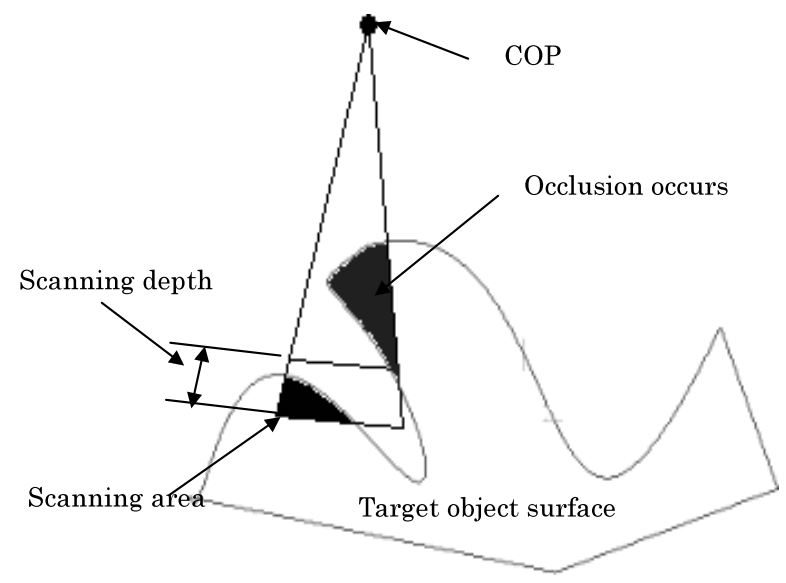

Fig. 10 Occlusion occurs outside viewing volume

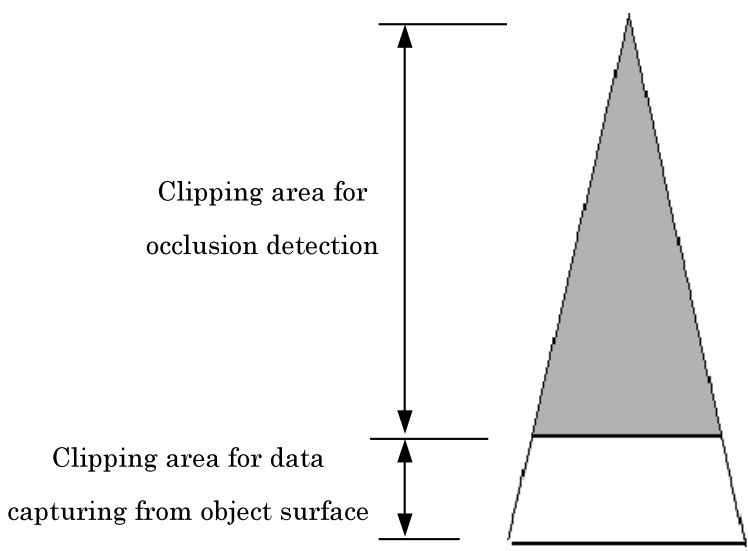

Fig. 11 Occlusion solution can be detected.

\section{Planning Scanning Path}

Based on the functionality of the simulator as a part of the planning system we prototype a planning system. So the functionality of the planning system is not sufficient to be applied to a real problem at this moment. A hierarchical planning algorithm is used which is divided into two levels, path planning and sensor planning. Sensor planning is discussed in subsection 5.2, while path planning in subsection 5.3. As a basis for these two levels of planning, subsection 5.1 discusses the cell decomposition. The workflow is described in the following.

Step1. According to the specification of sensor model process, the workspace is decomposed into cells. Then calculate the triangles that exist in each $C_{i}$ cell.

Step2. Generate the sensing plans for each cell by defining its sensor configurations (position \& orientation).

Step3. For each cell, perform simulation to obtain the triangle-IDs and position data.

Step4. For each cell, perform collision detection and occlusion test.

Step5. Process the path planning according to sensor configuration data from sensor planning, and export the generated paths to real scanning machine. These paths also guide the motion simulation of links and sensor model with inverse kinematics method.

\subsection{Cell decomposition}

We decompose the workspace to a cubic cells arranged in the $3 \mathrm{D}$ regular grids. We define scanning plan (sensor position and direction) for each cell. That is, for cell $i$, we determine scanning volume $F_{i}$. By denoting the cell region $R_{i}$, obviously we need to decide the cell size so as to satisfy $R_{i} \subseteq F_{i}$. In this research the cell sizes length $\times$ width $\times$ height are represented by parameters D1 $\times$ D $2 \times D 3$ as shown in Fig. 6 . The parameters D1, D2, and D3 are determined by the specification of sensor model as shown in Fig. 6. While the evenly sized cells are verified that can not guide good scanning results according to experiences in real industrial experiments. In order to solve this problem "wrap over" is usually adopted in the industry. Amount of "wrap over" is often represented as a ratio $a \%$ to the cell size and often determined by experience, as shown in Fig. 12. They may be different from $X$, $Y$ or $Z$-direction. The detail position of a cell is described with its corner coordinates $\left(s_{x j}, s_{y j}, s_{z j}\right)$ and $\left(e_{x j}, e_{y j}, e_{z j}\right)$.

After getting the cell position the next step is to search triangles inner or intersecting within a cell, assume cell count is $N c$ and triangle count is $N t$, if we search all triangles inside each cell, or search all cells for each triangle, there will cost $O(N c \times N t)$ calculating times. We give a quick way to locate cells according to $\mathrm{max} / \mathrm{min}$ coordinates of each triangle. Then sort triangles to each cell with a hash table, then the calculating cost will be limited 


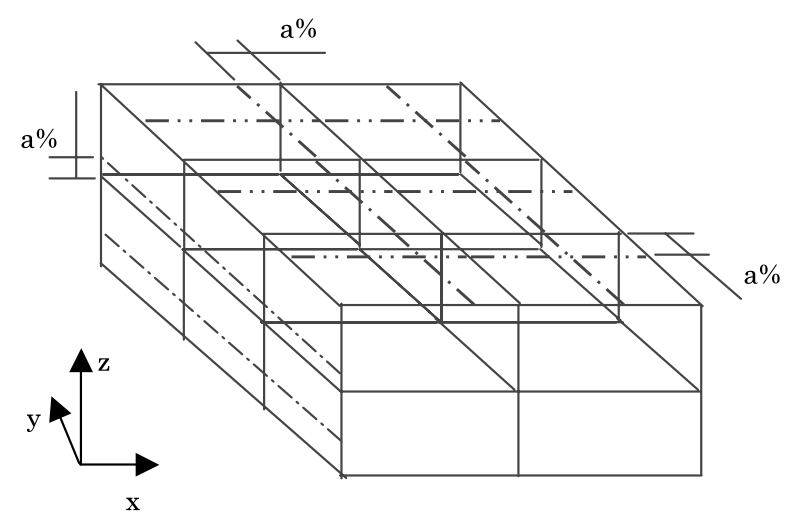

Fig. 12 Layered Z-map cells with wrap over

to $O(N t+N c)$.

\subsection{Sensor planning}

Take into account the various configurations of $3 \mathrm{D}$ scanning machines, we give a sensor planning for three kinds of machines: 3-axis, 4-axis and 6-axis. Sensor planning is to calculate the scanning position and direction $M_{i}=\left(C_{i}, \vec{V}_{i}\right)$ for each cell, and get a series of $M_{i}$ to scan the surface of the target object. If there is no triangle included in a cell, it is not necessary to calculate $M_{i}$ for this cell.

We calculate the average of the normal vectors of the triangles included in the cell and define its inverse as the viewing direction of $M_{i}$. The average normal of a cell is the area-weighted average of all the normal of the triangles inside the cell defined as follows:

$$
\vec{V}_{i}=-\frac{\sum_{j \in c e l l_{i}} \vec{N}_{j} \cdot A_{j}}{\sum_{j \in c e l l_{i}} A_{j}}
$$

where cell $i$ is a set of triangles included in the cell $i . \vec{N}_{j}$ is the normal of a triangle $j, A_{j}$ the area of the triangle $j$, and $V_{i}$ the view-direction of the cell.

To determine the view-port position (COP) we decide the weighted center of these triangles firstly according to the following equation.

$$
C_{i}^{\prime}=\frac{\sum_{j \in \text { cell }_{i}} T_{j} \cdot A_{j}}{\sum_{j \in \text { cell }_{i}} A_{j}}
$$

where $T_{i}$ is the center of a triangle, and $C_{i}^{\prime}$ the viewposition of the cell. Then we can easily get the view-port position COP according to Eq. (3).

$$
C_{i}=C_{i}^{\prime}+\left(d_{1}-\frac{d_{2}-d_{1}}{2}\right) \vec{V}_{i}
$$

According to the above equations the calculated view-position $C_{i}$ and view-direction $\vec{V}_{i}$ are adapted for 6axis machines only. It cannot be used for 3-axis and 4-axis machines directly. In this research we also implement our method for 3-axis and 4-axis.

For a 3 -axis machine the view-direction is fixed toward to minus $z$-direction, which is $(0,0,-1)$, so only the

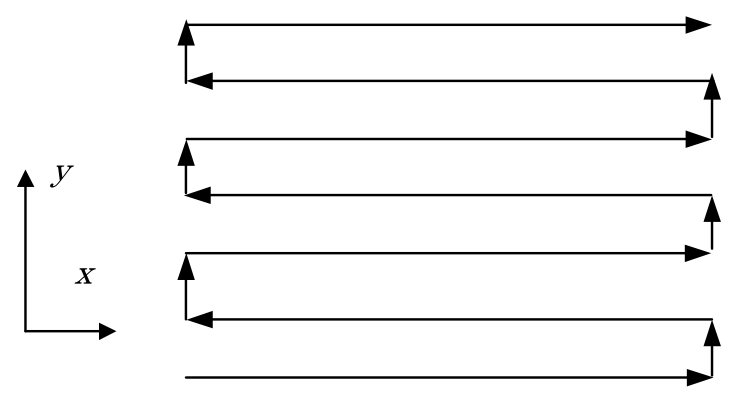

Fig. 13 Zigzag trajectory on a layer

view-position is need to be calculated with Eqs. (2) and (3). For 4-axis machine there is one rotational axis exists besides two translation axes $X Y Z$. The rotational axis is about $y$-axis in research, so the view-directions can only exist in $X Z$ plane, here we project the $\vec{V}_{i}$ to the $X Z$ plane firstly, then calculate view-position $C_{i}$ with Eqs. (2) and (3).

\subsection{Path planning}

After generating all the view-positions and viewdirection $M_{i}=\left(C_{i}, \vec{V}_{i}\right)$ for all the cells, then the scanning path can be generated by connecting these view-positions with properties of view-direction for each view-positions $M_{i}$. Techniques from NC machining and cutter-path generation could well be used in the path planning ${ }^{(19)}$. There are two types of connecting methods: one-way and zigzag. In this research we give a strategy to generate paths layer after layer, using a zigzag trajectory (Fig. 13) though there are more sophisticated methods.

The basic flow of this search is as follows:

1. Find view-positions of cells from first layer of decomposition cells.

2. Connect the view-positions along $x$-direction (or $y$-direction) until the end of this row and skip the empty view-positions that not any triangle exist in the cell, then link the next row along minus $x$-direction (or minus $y$ direction). Repeat this until the end of this layer.

3. Connect to the next layer of cells by the way of step 1 and 2. Repeat until the last layer.

\section{Implementation}

We implemented our system on a Pentium IV 1.5 GHz, windows 2000 machine with Visual $\mathrm{C}++6.0$. In this research we use a model for a press die to form sheet metal parts as our target object that comes from a real automobile car parts. We have three kinds of scanning machine configurations: 3-axis, 4-axis, and 6-axis. For these three kinds of manipulators we use the same sensor model whose capturing area is $50 \times 50$ pixels. The front clipping plane and back clipping plane are at the distance from COP $712 \mathrm{~mm}$ and $772 \mathrm{~mm}$ respectively. The scanning depth is $60 \mathrm{~mm}$ (D3). The wrap over percentage $a=20 \%$, which is recommended by experience. Figure 14 shows our implementation results. Figure 14 (a)-(c) shows the 


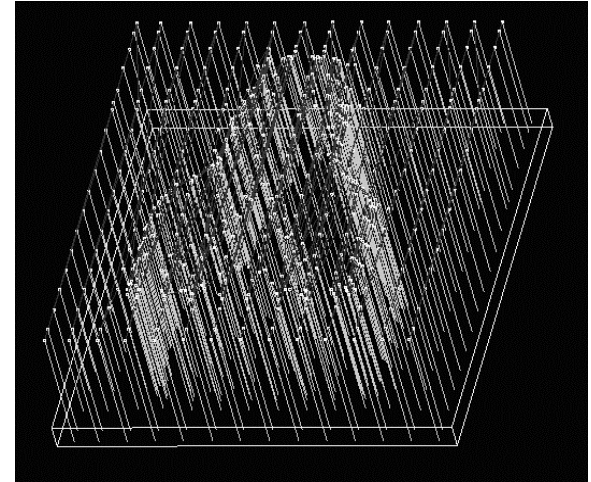

(a) Paths of the 3-axis scanning machine

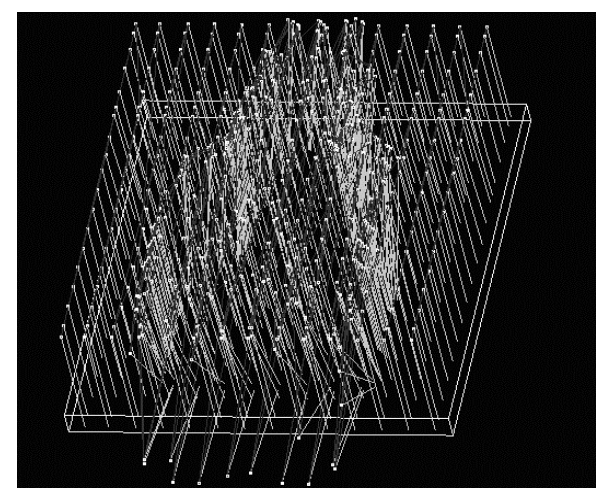

(b) Paths of the 4-axis scanning machine

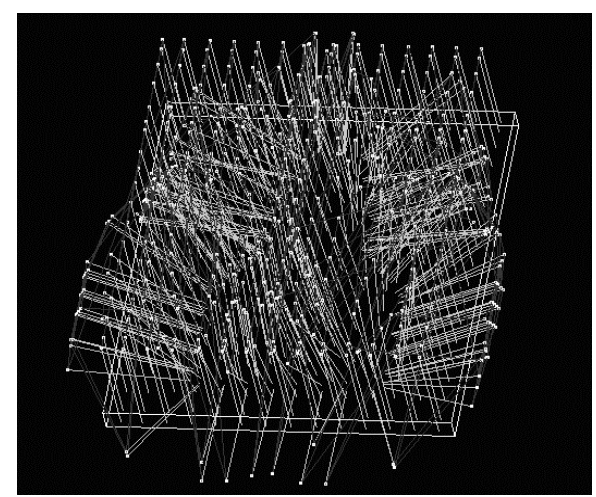

(c) Paths of the 6-axis scanning machine

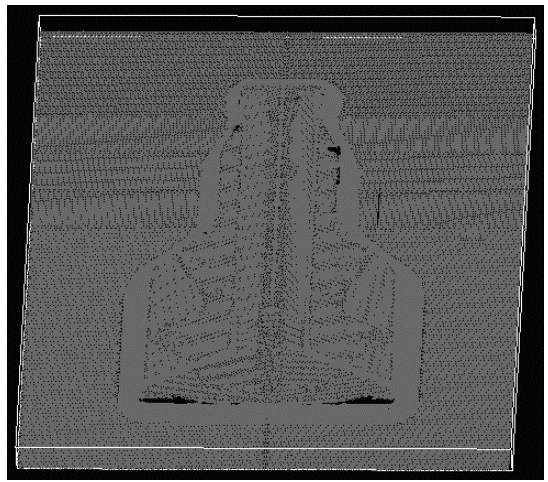

(d) Simulation result on 3-axis scanning machine

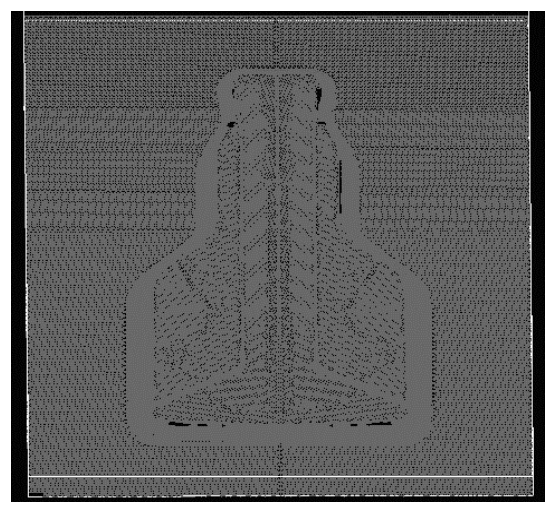

(e) Simulation result on 4 -axis scanning machine

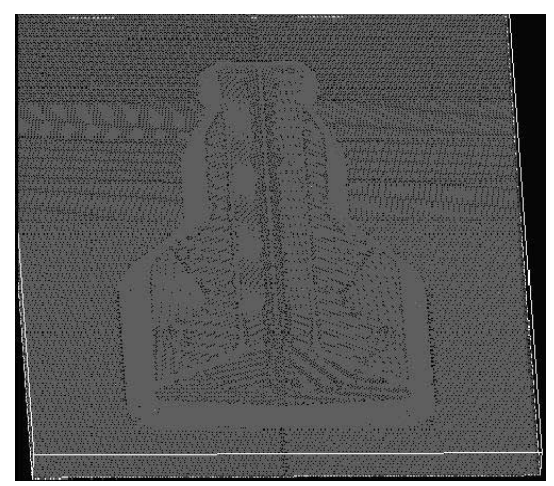

(f) Simulation result on 6-axis scanning machine

Fig. 14 Implementation results

generated scanning paths and view-directions for 3, 4, 6axis machine, and Fig. 14 (d) - (e) shows the implementation results. The calculation cost is listed in Table 1 .

At the same time of simulation we captured the triangle IDs, then the coverage of scanned areas by these three different kinds of manipulators are calculated. From the calculation result in Table 2 we can see that 3 -axis and 4-axis manipulators are not able to give the good planning result as compared to 6-axis one. It is shown that the higher the degree of freedom of the scanning machine, the wider the coverage of scanning area.
Table 1 Calculation statistics for implementation

\begin{tabular}{l|l}
\hline \multicolumn{1}{c|}{ Item } & \multicolumn{1}{c}{ Value } \\
\hline Cell-decomposition size $(\mathrm{mm})$ & $178 * 178 * 60$ \\
\hline Cell count & 1512 \\
\hline Cell-decomposition time (ms) & 0.19 \\
\hline Map Cells and triangles time(s) & 53.137 \\
\hline View-position and view-direction & 0.5 \\
calculation time (ms) & \\
\hline Zigzag path generation time (s) & 0.05 \\
\hline
\end{tabular}


Table 2 Coverage of three kinds of scanning machines

\begin{tabular}{c|l|l|l}
\hline & 3 -axes & 4 -axes & 6 -axes \\
\hline Coverage & $97.43 \%$ & $98.8 \%$ & $100 \%$ \\
\hline
\end{tabular}

\section{Conclusion}

In this paper we proposed an automatic model-based planning system for 3D surface scanning machine. Firstly we describe a multi-axis simulator with an OpenGL sensor model, where the geometric information of a CAD model is used. Then we describe how to detect collisions and occlusions for our simulation system. In order to improve calculating efficiency, we implement our algorithm based on a hardware-accelerated algorithm. We propose a rough path planning method in order to increase surface scanning coverage and reduce measuring time. The planned paths can be simulated with our scanning simulator, and can be exported to a real 3D scanning machine. Finally we implement our approach and verify the effectiveness of handling press parts. There still exist many future works. For example, aiming at handling complicated automotive parts model fine planning is our next research phase.

\section{Acknowledgements}

The authors would like to thank OPTON Company, Japan for their cooperation in this work.

\section{References}

( 1 ) Maver, J. and Bajcsy, R., Occlusions as a Guide for Planning the Next View, PAMI, Vol.15, No.5 (1993), pp.417-433.

( 2 ) Pudney, C.J., Surface Modelling and Surface Following for Robots Equipped with Range Sensors, Ph.D. Thesis, Univ. of Western Austrialia, Perth, (1994).

( 3 ) Whaite, P. and Ferrie, F.P., Autonomous Exploration: Driven by Uncertainty, PAMI, Vol.19, No.3 (1997), pp.193-205.

( 4 ) Connolly, C.I., The Determination of Next Best Views, CRA85, (1985), pp.432-435.

( 5 ) Massios, N.A. and Fisher, R.B., A Best Next View Selection Algorithm Incorporating a Quality Crite- rion, British Machine Vision Conference 1998, Sept., (1998), pp.780-789.

( 6 ) Pito, R., A Sensor Based Solution to the Next-BestView Problem, IEEE Int. Conf. on Robotics and $\mathrm{Au}-$ tomation, Aug., (1996), pp.941-945.

( 7 ) Grimson, W.E.L., Sensing Strategies for Disambiguating among Multiple Objects in Known Poses, MIT AI Memo, (1985).

( 8 ) Hutchinson, S.A. and Kak, A.C., Planning Sensing Strategies in Robot Work Cell with Multi-Sensor Capabilities, RA, Vol.5, No.6 (1989), pp.765-783.

(9) Xia, F. and Shub, C., CAD-Based Path Planning for 3-D Line Laser Scanning, Computer-Aided Design, Vol.31, No.7 (1999), pp.473-479.

(10) Sheng, W., Xi, N., Song, M. and Chen, Y., CADGuided Sensor Planning for Dimensional Inspection in Automotive Manufacturing, IEEE/ASME Transactions on Mechatronics, Vol.8, No.3 (2003), pp.372-380.

(11) Latombe, J.C., Robot Motion Planning, (1991), Kluwer Academic Publishers, Boston, MA.

(12) Segal, M. and Akeley, K., The OpenGL Grahics System: A Specification, Version 1.0, Silicon Graphics, (1992).

(13) Gottschalk, S., Lin, M. and Manocha, D., ObbTree: A Hierarchical Structure for Rapid Interference Detection, Computer Graphics, Proceedings of SIGGRAPH'96, Aug., (1996), pp.171-180. http://www.cs.unc.edu/geom/OBB/OBBT.html

(14) Angel, E., Interactive Computer Graphics: A TopDown Approach with OpenGL, ISBN 0-201-85571-2, (1997), Addison Wesley Longman, Inc.

(15) DeGarmo, E.P., Black, J.T. and Kohser, R.A., Materials and Processes in Manufacturing, (1988), Macmillan, New York.

(16) Canny, J., Collision Detection for Moving Polyhedra, PAMI, Vol.8, No.2 (1986), pp.200-209.

(17) Cameron, S., Collision Detection by Four-Dimensional Interscection Testing, Proceedings of International Conference on Robotics and Automation, (1990), pp.291-302.

(18) Garica-Alonso, A., Serrano, N. and Flaquer, J., Solving the Collision Detection Problem, IEEE Computer Graphics and Applications, Vol.13, No.3 (1994), pp.36-34.

(19) Choi, B.K., Sculpture Surface Machining, (1998), Kluwer Academic Publishers, Boston, MA. 